\title{
Robust Biometric Key Extraction Based on Iris Cryptosystem
}

\author{
Long Zhang ${ }^{1}$, Zhenan $\mathrm{Sun}^{2}$, Tieniu Tan ${ }^{2}$, and Shungeng $\mathrm{Hu}^{1,3}$ \\ ${ }^{1}$ Center for Intelligence Science and Technology Research, \\ Beijing University of Posts and Telecommunications, Beijing 100876, China \\ ${ }^{2}$ Center for Biometrics and Security Research, National Laboratory of Patten Recognition, \\ Institute of Automation, Chinese Academy of Sciences, Beijing 100190, China \\ ${ }^{3}$ China Netcom Group Labs, Beijing 100140, China \\ zl.bupt@gmail.com, \{znsun,tnt\}@nlpr.ia.ac.cn, \\ hushungeng@chinaunicom.cn
}

\begin{abstract}
Biometric cryptosystem can not only provide an efficient mechanism for template protection, but also facilitate cryptographic key management, thus becomes a promising direction in information security field. In this paper, we propose a robust key extraction approach which consists of concatenated coding scheme and bit masking scheme based on iris database. The concatenated coding scheme that combines Reed-Solomon code and convolutional code is proposed so that much longer keys can be extracted from the iris data, while the bit masking scheme is proposed to minimize and randomize the errors occur in the iris codes, making the error pattern more suitable for the coding scheme. The experiment results show that the system can achieve a FRR of $0.52 \%$ with the key length of 938 bits.
\end{abstract}

Keywords: Biometrics, cryptography, iris code, key binding, concatenated coding scheme, Reed-Solomon code, convolutional code, bit masking scheme.

\section{Introduction}

Biometrics provides a convenient and reliable way for user authentication. Compared with the traditional cryptosystem which identifies people based on passwords, biometric authentication has its natural advantages: the user's physiological or behavioral characteristics are something he/she was born with, they are unique, stable and portable, thus can overcome the shortage of the passwords which could be easily guessed or forgotten.

Generally, there are two phases in biometric system for user authentication: during enrollment phase, for each registered user, a biometric template is created and stored in the database; during authentication phase, a user to be authenticated present his/her biometric sample, the system then compares it to the templates, only a successful match can the user enter the system. In such a system, biometric templates are not stored in any encrypted form, attacks on the templates can pose a fatal threat to the whole system because unlike the password, compromised biometric templates cannot 
be revoked or reissued, once compromised, they are compromised forever [1]. In order to gain higher security level and more public acceptance of biometric system, an efficient biometric template protection scheme should be proposed.

On the other hand, traditional cryptosystem suffers from the key managing problem [2]: cryptographic keys are long and random in order to meet the secure requirement of the system, but they are hard to remember. Instead, the cryptographic keys are stored somewhere in the system and released based on other authentication mechanism such as passwords, which are neither convenient nor secure. Biometrics would be a good alternative, but different from passwords, biometric data are noisy. The existence of intra-user variation among multiple acquisitions of the same biometric identifier makes the cryptographic key extraction a challenging and interesting task.

Biometric cryptosystem, which takes the advantage of both biometrics and cryptography, is developed with the purpose that a repeatable cryptographic key can be extracted from the error-prone biometric data in an error-tolerant way. In such a system, the biometric component plays the role of user authentication while the extracted key can be used in any traditional cryptosystem. Under a careful design, biometric cryptosystem can not only provide an efficient mechanism for template protection, but also facilitate the key management, thus opens new applications in information security field. The existing biometric cryptosystems in the literature can be classified into three categories [1,2]: key release system, key binding system and key generation system. In this paper, we designed a key binding biometric cryptosystem based on iris database where error-control code is used to handle intra-user variation. The existing key binding methods have two main shortages: firstly, the cryptographic keys extracted from the biometric data are not long enough, which narrows the use of key binding method in more cryptographic applications; secondly, the error-correction capability of the error-control code is not strong enough to handle large intra-user variation of the real biometric data, which may result in poor recognition performance (measured by FRR and FAR). To solve these problems, for the former, we propose a concatenated coding scheme which combines the RS and convolutional codes to increase the key length, and for the latter, we propose a bit masking scheme which reduces the intra-user variations of iris codes as well as to randomize them to the acceptable level in accordance with the capability of the error correcting code.

The remaining sections are organized as follows. In section 2 , we give a brief review of the existing methods of biometric cryptosystem. Then we describe our proposed method, especially the concatenated coding scheme and the bit masking scheme in section 3. The experiment results and analysis are discussed in section 4 . Section 5 is the conclusion.

\section{Related Works}

There are mainly three approaches to implement a biometric cryptosystem: key release approach, key binding approach and key generation approach. The key release approach is the most straightforward way to combine biometrics and cryptography. Soutar [13] developed such a system where the biometric template and the key are stored together as a user record in an encrypted form. During verification, the biometric subsystem decrypt the user record and performs biometric matching, and only after a successful match will the cryptographic key be released to the security subsystem. 
Although the segregation between verification process in the biometric subsystem and the rights and privileges which the user is assigned by the security subsystem will help the revocation of a user's rights and privileges, the exposure of biometric template during verification would make the system vulnerable to attacks. The key generation approach, on the other hand, may be the most difficult and attractive method with the purpose of generating a cryptographic key directly from the biometric data. Dodis et al. [10] proposed the fuzzy extractors to solve the problem. The secure sketch is firstly constructed in order to recover the biometric data to the template, and then the fuzzy extractor is built based on the secure sketch where a strong extractor is used to extract a uniformly random key from the biometric template. The drawback of this approach is that the cryptographic key cannot be revoked or reissued because the same biometric template always generates the same key.

The key binding approach provides a feasible way to generate uniformly distributed random keys from the biometric data as well as to protect biometric template securely. Through this approach, a random key is chosen for a special user and then bound with his/her biometric template to form public information, e.g. helper data, then the biometric sample which is close enough to the template will be able to extract the key from the helper data.

Davida et al. [3] were among the first to apply error-control code to construct an off-line biometric authentication system. Given a canonical iris template, check bits are computed using a selected error-control code to form a codeword, then the check bits along with the hashed codeword are stored as the helper data. If user's biometric sample is close to the template to some extent, the check bits will eliminate the intrauser variations and make the authentication success, meanwhile, the hash function secures the template. Digital signature is also used to bind the biometric data to user attributes. To make the method more resilient and secure, Jules and Wattenberg [4] made some improvements and proposed fuzzy commitment scheme. Instead of regarding the template as the information bits, this scheme treats the template itself as a corrupt codeword, which can commit the cryptographic key in a fuzzy way. Along this line, there are many implementations to realize such integration of biometrics and cryptography. Hao et.al [5] designed a key binding system based on iris database using two-layer error-control code that combines Reed-Solomon and Hadamard codes. The length of the key extracted from the iris code is 140 bits with acceptable recognition performance, and can meet the requirement of key length in some cryptographic applications (such as 128-bit AES). Tuyls et al. [6] proposed the reliable components scheme based on fingerprint database in order to reduce the intra-user variation. The fuzzy vault scheme proposed by Jules and Sudan [7] may be regarded as an order-invariant version of [4] which is based on set difference. The secret, e.g. the cryptographic key is hidden in a selected polynomial as its coefficient, the vault is then constructed with the help of both genuine points and chaff points. The sample feature set which overlaps the template feature set substantially will be able to reconstruct the polynomial, and thus extract the key. Clancy et al. [8] and Uludag et al. [9] developed such a system based on fingerprints. Draper et al. [11] applied the distributed source coding to ensure the secure storage of fingerprint template. Besides, Itakura and Tsujii [12] proposed a method to apply biometric cryptosystem to PKI environment where the cryptographic key extracted from the biometric data is used as the user private key. 
The feature representation of the selected biometric identifier determines which approach should be chosen to design a biometric cryptosystem. If the feature set contains deletions and insertions of its element such as fingerprint, the fuzzy vault scheme is a good choice. And since the iris code is represented as a fix length string, we follow the method proposed in [4] in this paper.

\section{Our Proposed Method}

In this section, we present the detailed design of our iris cryptosystem, where the verification mode is considered. The iris code is a fixed length feature vector representation of the iris data which can be compared directly using Hamming distance. And the recognition performance of the iris cryptosystem is measured by the False Rejection Rate (FRR) and the False Acceptance Rate (FAR). The FRR is the error rate that the cryptographic key is not correctly extracted with the iris data from the same person, whereas the FAR is the error rate that the cryptographic key is mistakenly extracted with the iris data from different persons. There is always a tradeoff between FRR and FAR where the error-correction capability is the threshold. The system can adjust this threshold to meet different requirements of the applications.

Figure 1 shows the block diagram of the proposed iris cryptosystem. The enrollment phase is comprised of three steps: firstly, the bit masking component is performed to add the masking bits to original iris code $I_{T}$ to form a masked iris string $I_{T M} \in\{0,1\}^{n}$, where $n$ denotes the length of the iris code; secondly, a uniformly distributed key $K \in\{0,1\}^{k}$ is randomly generated for a special user, and then encoded into a codeword $C \in\{0,1\}^{n}$ through the concatenated encoding component; thirdly, the helper data $P$ that binds the cryptographic key and the iris template together is generated by $P=C \oplus I_{T M}$, where $\oplus$ denotes bitwise XOR. The information about neither the iris template nor the cryptographic key is revealed to the adversary given the helper data $P$.

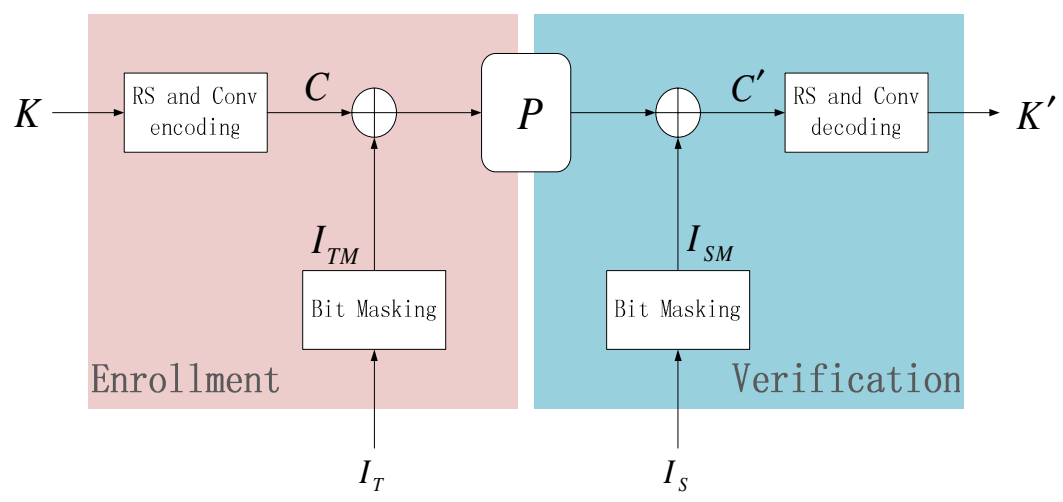

Fig. 1. Overview of the iris cryptosystem 
Accordingly, the verification phase comprises three steps: firstly, the system captures and extracts the sample iris code $I_{S}$ from the user to be verified, and then masked it in the same way as it did during the enrollment phase to form $I_{S M} \in\{0,1\}^{n}$; secondly, codeword $C^{\prime} \in\{0,1\}^{n}$ is calculated based on the helper data, $C^{\prime}=P \oplus I_{S M}=C \oplus\left(I_{T M} \oplus I_{S M}\right)$; and thirdly, cryptographic key $K^{\prime} \in\{0,1\}^{k}$ is extracted through the concatenated decoding, and if the iris codes $I_{T M}$ and $I_{S M}$ are close enough to each other, e.g. they are from the same person, the exacted key $K^{\prime}$ will be identical to $K$, which indicates a successful verification. As shown in figure 1 , the concatenated coding scheme and the bit masking scheme are the main factors which directly determine the recognition performance of the iris cryptosystem.

\subsection{Concatenated Coding Scheme}

Concatenated coding is a particular and efficient method for constructing long codes from short codes, as well as for increasing the error-correction capability of the errorcontrol codes. Applying the concatenated coding scheme to the iris cryptosystem, much longer keys can be embedded into the iris templates, which can then be extracted for legitimate users and used in more cryptographic applications.

There are two types of errors between different iris codes from the same eye: the random errors mainly caused by the precision of the acquisition device, and the burst errors mainly caused by undetected eyelashes and specular reflections. Based on the error characteristic of iris codes, Hao et.al [5] designed a two-layer concatenated coding scheme, in which Hadamard code is firstly used to correct the random errors, and then Reed-Solomon code is used to correct the burst errors.

The concatenated coding scheme in this paper handles the errors in iris codes in a different way. The burst errors are firstly transformed into random ones using the bit masking scheme which will be detailed in section 3.2. Then convolutional code and Reed-Solomon code are concatenated to control the errors. Convolutional code is chosen as the inner code because it is suitable to correct random errors, and compared with block coding, convolutional coding with the same code rate can achieve a better error-correction performance. Since burst errors often occur during the decoding phase of the convolutional code, Reed-Solomon code is used as its outer code to correct them at block level. The concatenated coding scheme of convolutional code and Reed-Solomon code is widely used in digital communication field to control the channel noise, but never had it been used in biometrics. In this paper, we will show that this coding scheme is an efficient way to bridge the gap between the fuzziness of the iris codes and the exactness of the cryptography.

Reed-Solomon code is a sub type of BCH code which is non-binary. The ReedSolomon code we choose to design the cryptosystem is defined on the Galois Field $G F\left(2^{m}\right)$, where $m$ denotes the size of a block. The code is denoted as $R S\left(n_{1}, k_{1}, t\right)$, where $k_{1}$ stands for the block number before encoding, and $n_{1}$ stands for the block number after encoding. The error-correction capability is determined by $t$, which is the number of error blocks that can be corrected, and $n_{1}-k_{1}=2 t$ must be specified. During verification, Berlekamp-Messey algorithm is used for decoding.

Different from block code, convolutional code operates on serial data where its encoder comprises a group of memory registers. The convolutional code can be denoted as $\operatorname{Conv}\left(n_{2}, k_{2}, v\right)$, where $k_{2}$ stands for the bit length of the encoder input stream, and 
$n_{2}$ stands for the encoder output stream. The error-correction capability is determined by $v$, which is the number of registers of the encoder. During verification, Viterbi algorithm is used for decoding.

The parameters of our design are set as follows. The length of the iris codes $n$ is fixed at 4096 bits. The code rate of convolutional code $R_{2}=k_{2} / n_{2}=1 / 4$, and the register number $v=6$, which indicates that there should be a tail of 6 zero-bits to flush the registers after convolutional encoding. The block size $m$ of the RS code is 8 bits, then the length of user-specific key can be calculated.

$$
k=k_{1} \times m-v=\left(\frac{n_{1}}{m}-2 t\right) \times m-v=\left(\frac{n \times R_{2}}{m}-2 t\right) \times m-v=(128-2 t) \times 8-6
$$

The number of blocks that can be corrected by the Reed-Solomon code $t$ is the threshold of the iris cryptosystem. By adjusting the value of $t$, the key length and the system recognition performance can be changed in light of requirements for different applications.

\subsection{Bit Masking Scheme}

In iris cryptosystem, the errors of iris codes are always too many to be handled by the error-control code, which is the main factor that degrades the system recognition performance. Moreover, there exist both random errors and burst errors in iris codes from the same eye, which is not a suitable error pattern for our coding scheme. If some positions of the iris codes to be compared can be replaced with the same bits, then the Hamming distance can be reduced. And if these positions are selected uniformly, the burst errors will be randomized. Bit masking scheme is proposed to deal with the errors in this way.

During enrollment, the template iris code $I_{T}=\left\{I_{T i}\right\}, i=1,2, \ldots, n$, where $I_{T i}$ denotes the $i$-th bit of the template code, is uniformly masked by the masking bits $M_{T}=\left\{M_{T i}\right\}$, where $M_{T i}$ denotes the masking bit at the $i$-th position of the template code. Then during verification, the sample iris code $I_{S}=\left\{I_{S i}\right\}, i=1,2, \ldots, n$, where $I_{S i}$ denotes the $i$ th bit of the sample code, is masked at the same positions by the masking bits $M_{S}=\left\{M_{S i}\right\}$, where $M_{S i}$ denotes the masking bit at the $i$-th position of the sample code. The masking bits at the same positions in both the template and the sample codes should keep the same, e.g. $M_{T i}=M_{S i}$.

Furthermore, we define the bit masking rate, denoted as $R_{M}$, to describe the extent to which the iris codes are masked. For example, $R_{M}=1 / 4$ means the iris codes are masked at the positions $i=4 j, j=1,2, \ldots, n / 4$. Let $I_{T M}=\left\{I_{T M i}\right\}, i=1,2, \ldots, n$ denotes the masked template code, and $I_{S M}=\left\{I_{S M}\right\}, i=1,2, \ldots, n$ denotes the masked sample code, they are computed as follows:

$$
I_{T M i}=\left\{\begin{array}{ll}
I_{T i} & , i \neq 4 j \\
M_{T i} & , i=4 j
\end{array}, \quad I_{S M i}= \begin{cases}I_{S i} & , i \neq 4 j \\
M_{S i} & , i=4 j\end{cases}\right.
$$

Since $M_{T i}=M_{S i}$, we can get $I_{T M i} \oplus I_{S M i}=0, i=4 j, j=1,2, \ldots, n / 4$.Similarly, $R_{M}=1 / 2$ means the iris codes are masked at the positions $i=2 j, j=1,2, \ldots, n / 2$, and we get $I_{T M i} \oplus I_{S M i}=0, i=2 j, j=1,2, \ldots, n / 2 . R_{M}=0$ stands for the situation that the iris codes are acquired without bit masking scheme. 
Obviously, the bit masking scheme can reduce the errors between iris codes, as well as to randomize them. After the iris codes are masked, the proposed concatenated coding scheme can be used to control errors efficiently. But the bit masking rate $R_{M}$ should be carefully selected. That is because with the increase of the bit masking rate, both the intra-user variations and inter-user variations fall down. If $R_{M}$ is set too low, the intra-user variations may not be sufficiently reduced, which results in high FRR. On the other hand, if $R_{M}$ is set too high, the inter-user variations may decrease, which results in high FAR. We suggest that the bit masking rate should be set in accordance with the error-correction capability of the coding scheme.

\section{Experimental Results and Analysis}

The iris database in the experiment consists of the iris codes from 128 different eyes, and each with 3 measurements. The length of each iris code is 4096 bits. Figure 2(a) shows the Hamming distance distribution of these iris codes. The intra-class Hamming distances are less than $30 \%$, while the inter-class Hamming distances are more than $40 \%$. Then the bit masking schemes with the rates $R_{M}=1 / 4$ and $R_{M}=1 / 2$ are applied to the iris codes respectively, the Hamming distance distributions are changed accordingly as shown in Figure 2(b) and Figure 2(c). With the increase of the masking rate, the curves of intra-class distribution and inter-class distribution become sharper and get closer to each other.

The database contains 3 iris codes per person, and we randomly choose one of them during enrollment. To calculate FRR, we use the remaining two iris codes during verification, thus totally $128 \times C_{3}^{2}=384$ comparisons should be made. To calculate FAR, we use the iris codes from the other persons during verification, which results in totally $\left(128 \times C_{3}^{1}\right) \times\left(127 \times C_{3}^{1}\right) / 2=73152$ comparisons.

The Reed-Solomon code chosen in our scheme is the shortened code on Galois Field $G F\left(2^{8}\right)$, and the convolutional code is the optimal code with $1 / 4$ code rate given in [14]. The concatenated coding scheme is performed on the iris codes with different bit masking rates $R_{M}=0, R_{M}=1 / 4$ and $R_{M}=1 / 2$, respectively. With the increase of bit masking rate, the errors occur in the iris codes are reduced and become more random, which can be corrected more easily by the concatenated code. As a result, the FRR decreases while the FAR increases. Figure 3(a) shows the FRR curves for different masking rate, and since the FAR values with the masking rate $R_{M}=0$ and $R_{M}=1 / 4$ identically equal to 0 at all the thresholds, we do not show the comparison of the FAR curves. Generally, smaller value of the threshold $t$ results in longer keys with lower FAR but higher FRR, and vice versa. In our design, $R_{M}=1 / 2$ is a suitable value and the FAR and FRR curves are shown in Figure 3(b). The system can extract a user-specific key of 938 bits, with $F R R=0.52 \%$ and FAR $=0$. The key extracted from the iris codes is much longer than the existing methods in literatures $[5,6,8,9]$. And the key can meet many cryptographic applications, such as 128-bit, 192-bit and 256-bit AES. Besides, in the public key cryptosystem, the extracted key 
from the iris data can also be used as the user's private key, from which the publickey certificate can be derived. The certificate with a PKI signature can then be used for remote authentication in the network environment.

By our proposed key binding iris cryptosystem, the iris database should meet the following two requirements to ensure the robustness of long biometric key extraction. Firstly, the iris code itself should be long, and if the iris code is not long enough, we can insert the same bits uniformly in both template and sample codes instead of the bit masking scheme, which can not only increase the length of the iris code, but also randomize the errors. Secondly, the captured iris images should be of high quality, which is helpful for our scheme to achieve a better recognition performance.

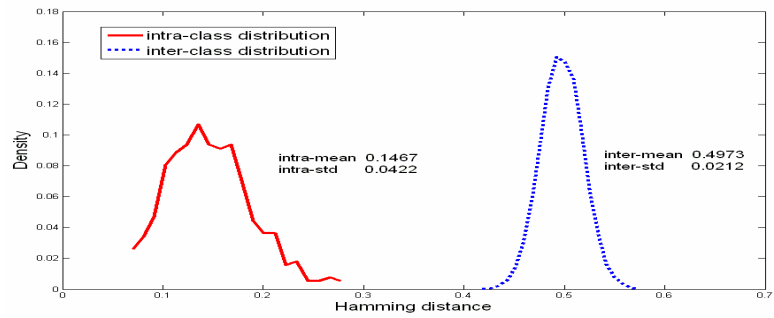

(a)

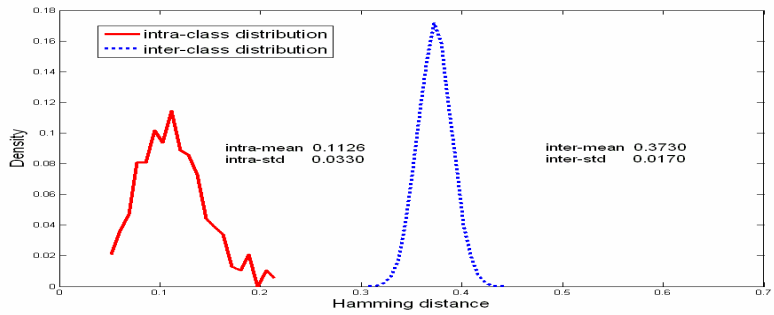

(b)

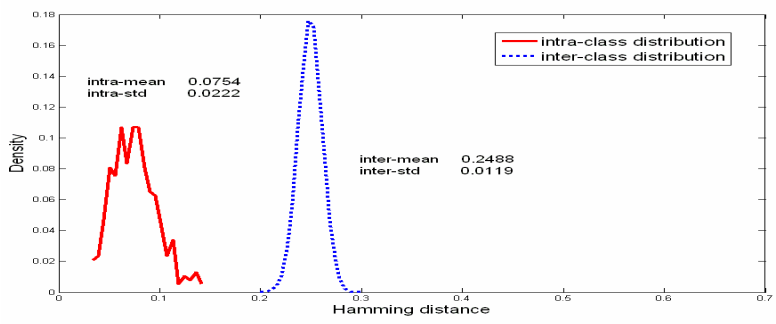

(c)

Fig. 2. Hamming distance distributions: (a) with bit masking rate $R_{M}=0$; (b) with bit masking rate $R_{M}=1 / 4 ;$ (c) with bit masking rate $R_{M}=1 / 2$ 


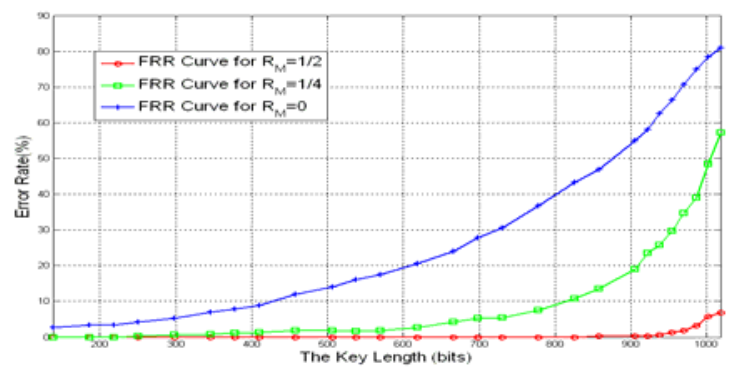

(a)

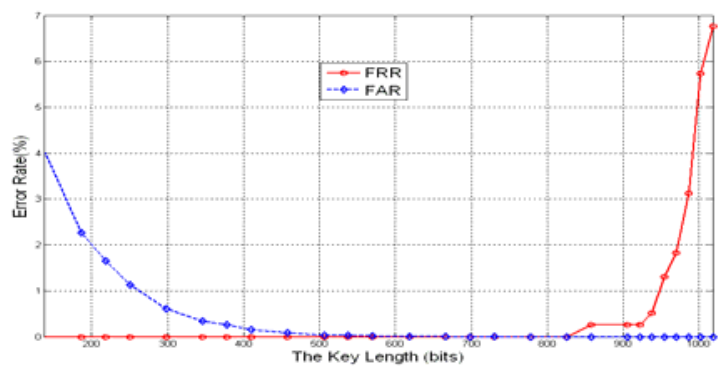

(b)

Fig. 3. Recognition performance of proposed iris cryptosystem: (a) FRR curves for different masking rates; (b) FAR and FRR curves for $R_{M}=1 / 2$

\section{Conclusions}

In this paper, concatenated coding scheme and bit masking scheme are proposed to construct an iris cryptosystem. The concatenated coding scheme is proposed in order to embed long keys into the iris data. We apply the concatenated code that combines Reed-Solomon code and convolutional code, which is a prevalent channel coding technique in digital communication field, to control the errors in the iris codes. Then the bit masking scheme is proposed to minimize and randomize the errors to make the error pattern more suitable for the coding scheme. The experiment results prove that our iris cryptosystem is robust and efficient since much longer keys can be extracted from the iris data while the system recognition performance remains acceptable. The iris cryptosystem achieves a FRR of $0.52 \%$ with the key length of 938 bits.

Acknowledgments. This work is supported by National 973 Program Project (Grant No. 2004CB318100), National Natural Science Foundation Projects (Grant No. 60736018, 60335010, 60702024, 60723005), and National 863 Program Projects (Grant No. 2006AA01Z193, 2007AA01Z162). 


\section{References}

1. Jain, A.K., Nandakumar, K., Nagar, A.: Biometric Template Security. EURASIP Journal on Advances in Signal Processing 2008, Article ID 579416, 17 (2008), doi:10.1155/2008/ 579416

2. Uludag, U., Pankanti, S., Prabhakar, S., Jain, A.K.: Biometric cryptosystems: issues and challenges. Proceedings of the IEEE 92(6), 948-960 (2004)

3. Davida, G.I., Frankel, Y., Matt, B.J.: On enabling secure applications through off-line biometric identification. In: Proceedings of the IEEE Computer Society Symposium on Research in Security and Privacy, Oakland, Calif, USA, pp. 148-157 (May 1998)

4. Juels, A., Wattenberg, M.: A fuzzy commitment scheme. In: Proceedings of 6th ACM Conference on Computer and Communications Security (ACM CCS 1999), Singapore, pp. 28-36 (November 1999)

5. Hao, F., Anderson, R., Daugman, J.: Combining crypto with biometrics effectively. IEEE Transactions on Computers 55(9), 1081-1088 (2006)

6. Tuyls, P., Akkermans, A.H.M., Kevenaar, T.A.M., Schrijen, G.-J., Bazen, A.M., Veldhuis, R.N.J.: Practical biometric authentication with template protection. In: Kanade, T., Jain, A., Ratha, N.K. (eds.) AVBPA 2005. LNCS, vol. 3546, pp. 436-446. Springer, Heidelberg (2005)

7. Juels, A., Sudan, M.: A fuzzy vault scheme. In: Proceedings of the IEEE International Symposium on Information Theory, Piscataway, NJ, USA, p. 408 (June-July 2002)

8. Clancy, T., Lin, D., Kiyavash, N.: Secure smartcard-based fingerprint authentication. In: Proceedings of the ACMSIGMM Workshop on Biometric Methods and Applications, Berkley, Mich, USA, pp. 45-52 (November 2003)

9. Uludag, U., Pankanti, S., Prabhakar, S., Jain, A.K.: Fuzzy vault for fingerprints. In: Kanade, T., Jain, A., Ratha, N.K. (eds.) AVBPA 2005. LNCS, vol. 3546, pp. 310-319. Springer, Heidelberg (2005)

10. Dodis, Y., Reyzin, L., Smith, A.: Fuzzy extractors: how to generate strong keys from biometrics and other noisy data. In: Cachin, C., Camenisch, J.L. (eds.) EUROCRYPT 2004. LNCS, vol. 3027, pp. 523-540. Springer, Heidelberg (2004)

11. Draper, S.C., Khisti, A., Martinian, E., Vetro, A., Yedidia, J.S.: Using distributed source coding to secure fingerprint biometrics. In: Proceedings of the IEEE International Conference on Acoustics, Speech and Signal Processing (ICASSP 2007), Honolulu, Hawaii, USA, vol. 2, pp. 129-132 (April 2007)

12. Itakura, Y., Tsujii, S.: Proposal on a multifactor biometric authentication method based on cryptosystem keys containing biometric signatures. International Journal of Information Security, 288-296 (2005)

13. Soutar, C.: Security Considerations for the Implementation of Biometric Systems. In: Ratha, N., Bolle, R. (eds.) Automatic Fingerprint Recognition Systems, pp. 415-431. Springer, Heidelberg (2004)

14. Lin, S., Costello, D.J.: Error Control Coding Second Edition. China Machine Press, Beijing (2007) 